\title{
Cenário da produção conjunta entre Brasil e Espanha na Física de Altas Energias (1992-2013)
}

\author{
Scenario of scientific output of Brazil and Spain in the High Energy Physics (1992-2013)
}

Gonzalo Rubén Alvarez

Doutorando no Programa de Pós-graduação em Comunicação e Informação da Universidade Federal do Rio Grande do Sul - UFRGS.

E-mail: gonzalorubenalvarez@gmail.com

\author{
Ana Isabel Bonilla-Calero \\ Doutora pela Universidade Carlos III de Madrid. \\ Funcionária da Agencia Nacional de Evaluación de la Calidad y Acreditacion (ANECA) \\ E-mail: anabel.bonilla@gmail.com
}

\begin{abstract}
María Luisa Lascurain-Sánchez
Doutora em Documentación pela Universidad Carlos III de Madrid. Profesora titular do Departamento de Biblioteconomía y Documentación da Universidad Carlos III de Madrid. E-mail: mlascura@bib.uc3m.es
\end{abstract}

Samile Andrea de Souza Vanz Doutora em Comunicação e Informação pela Universidade Federal do Rio Grande do Sul - UFRGS. Professora adjunta do Departamento de Ciências da Informação e do Programa de Pós-graduação em Comunicação e Informação da Universidade Federal do Rio Grande do Sul - UFRGS.

E-mail: samilevanz@terra.com.br

\section{Resumo}

Este estudo bibliométrico analisa as características da produção conjunta entre Brasil e Espanha na Física de Altas Energias (FAE) a partir dos artigos publicados em revistas indexadas pela Web of Science (WoS) no período de 1983-2013. Indicadores de produção e colaboração foram utilizados na caracterização da área abordada. Os resultados mostram que a taxa de crescimento da colaboração multilateral (Brasil, Espanha e outros países) é superior ao crescimento individual da produção científica de cada país. Instituições estatais como a brasileira Rede Nacional de Física de Altas Energias (RENAFAE) e a espanhola Consejo Superior de Investigaciones Científicas (CSIC) tiveram papel fundamental para a promoção e coordenação das colaborações internacionais de ambos os países. A colaboração multilateral favorece as publicações em revistas Q1 e o incremento do número de citações. A colaboração bilateral em FAE não traz benefícios suficientes em termos de impacto. No entanto, esse tipo de colaboração propicia o compartilhamento de infraestrutura e conhecimentos científicos a partir de iniciativas e convênios de cooperação. Na FAE, a colaboração internacional é fundamental, pois melhora os indicadores quanto maior for o número de países envolvidos. No entanto, a participação do Brasil e da Espanha nas redes multinacionais não implica uma marcada especialização.

Palavras-chave: Produção científica. Colaboração Científica. Brasil. Espanha. Física de Altas Energias.

\begin{abstract}
This study analyzes the characteristics of the scientific output of Brazil and Spain in the High Energy Physics (HEP). The corpus of research is comprised of articles published in journals indexed by Web of Science (WoS) in the 1983-2013 period. Bibliometric indicators of output and collaboration were used in the characterization of the area. The results show that the growth rate of multilateral collaboration (Brazil, Spain and other countries) is greater than the individual growth of scientific production in each country. National institutions such as the Brazilian Rede Nacional de Física de Altas Energias (RENAFAE) and the Spanish Consejo Superior de Investigaciones Cientificas (CSIC) played a key role in the promotion and coordination of international collaborations in both countries. Multilateral cooperation favors publications in Q1 journals and the citations. Bilateral cooperation in HEP does not bring enough benefits in terms of impact. However, this type of collaboration provides the infrastructure sharing and scientific knowledge. In HEP, international collaboration is crucial because a greater number of countries involved results in better indicators. However, the participation of Brazil and Spain in multinational networks does not imply a marked specialization.
\end{abstract}

Keywords: Scientific output. Scientific collaboration. Brazil. Spain. High Energy Physics.

InCID: R. Ci. Inf. e Doc., Ribeirão Preto, v. 8, n. 1, p. 81-99, mar./ago. 2017.

DOI: 10.11606/issn.2178-2075.v8i1p81-99 


\section{Introdução}

Desde o advento da 'Grande Ciência', a colaboração internacional em áreas como a Física de Altas Energias (FAE) tem aumentado significativamente como consequência da complexidade e custo dos experimentos. De acordo com Chaves et al. (2002), a colaboração internacional atende sempre a assuntos da mais avançada vanguarda da Ciência, sendo essa a característica principal que justifica a sua própria existência. Na FAE, os fatores econômicos, vinculados aos altos custos dos equipamentos, têm sido um dos motivos essenciais da colaboração científica. Para a viabilização dos experimentos, o campo estudado requer a utilização de instrumentos caros como são os aceleradores de partículas, além da participação de especialistas de diversas áreas científicas tais como Engenharia, Matemática, Ciência da Computação e Geociências (BORDONS; GÓMEZ, 2000; VANZ; STUMPF, 2010). Segundo Leta e Cruz (2003), a redução de custos, a complexidade das pesquisas e a multidisciplinaridade são provavelmente os aspectos que mais estimulam a colaboração internacional. A FAE, as Ciências Espaciais, a Astrofísica e a Física Nuclear são exemplos de áreas que requerem uma gama de conhecimentos e o acesso a recursos consideráveis, em termos de pessoal e finanças, que se situam além das possibilidades econômicas de uma única nação (BRAUN et al., 1992).

Na Suíça, o The European Organization for Nuclear Research (CERN), é um distinguido exemplo de colaboração, destacando-se como o maior centro de pesquisa internacional para a realização de experimentos dentro da Física e por ser considerado como uma iniciativa que busca a construção de ligações mais fortes entre as nações (KATZ; MARTIN, 1997; DUARTE, 2008; LUUKKONEN et al., 1993). Ainda, existem outros motivos que encorajam os pesquisadores a trabalhar em colaboração, sendo eles: possibilidade de acesso a equipamentos especiais; recebimento de treinamento para desenvolvimento de habilidades; incremento da produtividade; avanço na carreira profissional; aquisição de novos conhecimentos (MEADOWS, 1999; MALTRÁS BARBA, 2003; GLÄNZEL; SCHUBERT, 2004; WAGNER, 2006). A ampliação da visibilidade dos resultados científicos também é um fator que estimula a colaboração, tendo em vista que os estudos de citação demonstram a correlação positiva entre o número de autores internacionais e número de citações recebidas, mesmo quando são eliminadas as autocitações.

Embora a colaboração internacional e o compartilhamento de infraestrutura, laboratórios e ideias em áreas da Big Science como a FAE seja uma realidade forte (BRAUN et al., 1992), é evidente que a contribuição de cada país não é homogênea. Por isso, a 
participação em grandes redes nem sempre pode ser considerada isoladamente como um indicador do grau de desenvolvimento de um país, já que um grupo pode estar colaborando de maneira 'assimétrica' com outros mais desenvolvidos, a fim de complementar as suas capacidades (MOURA et al., 2015). Por esse motivo, a contribuição de um país na colaboração internacional não é fácil de ser analisada. Meadows (1999) ressalta que as publicações de autoria múltipla oferecem alguns problemas para a definição de produtividade e questiona a maneira de como devem ser contabilizados os artigos para cada autor e o grau de contribuição dos mesmos no paper.

Os fatores que influem no tipo de colaboração entre os países são diversos: históricos, geográficos, políticos, socioeconômicos, linguísticos e referentes ao tamanho. Por exemplo, sabe-se que o grau de colaboração internacional de um país é inversamente proporcional ao seu tamanho científico, devido a dificuldade dos pesquisadores para encontrar colaboradores especialistas em seu próprio país, o que os leva a recorrer a esfera internacional.

Estudos de colaboração "bilateral” (dois países) são importantes já que permitem definir com maior precisão qual é a contribuição real de cada parte (DE FILIPPO; BARRERE; GÓMEZ, 2010). Os autores citados destacam que, para entender o peso dessa cooperação, é necessário conhecer as particularidades da atividade científica de cada um dos parceiros envolvidos e especificar seus padrões de produção e colaboração.

A escolha da FAE para um estudo de colaboração bilateral se justifica pelo fato de ser uma área caracterizada pelas grandes colaborações como consequência do custo e da complexidade das pesquisas experimentais. Em termos de benefícios sociais, a FAE contribui intensamente para áreas como a Medicina (desenvolvimento de tomógrafos e terapia de radiação para diagnóstico e tratamento do câncer); para a Tecnologia da Informação \& Comunicação (criação da World Wide Web1 como um sistema de processamento, transmissão e armazenamento de grandes volumes de dados); para o Meio Ambiente e Energia (produção de energia renovável ou "limpa" a partir do aproveitamento da grande quantidade de energia liberada pelas antipartículas e antimatéria nas suas interações) (SANTORO; NOVAES, 2003; LEFEVRE, 2007).

\footnotetext{
1 Sistema de servidores Internet que utiliza como protocolo principal o HyperText Transfer Protocol (HTTP) (MARÇULA; BENINI FILHO, 2005). Criada em 1989 e apresentada por Tim Berners Lee para o CERN em 1991, a World Wide Web surgiu com a finalidade de permitir que os pesquisadores da FAE pudessem acessar e compartilhar informações acerca dos resultados das investigações científicas.
} 
Este estudo visa caracterizar a produção conjunta entre Brasil e Espanha por meio de artigos escritos por autores de ambos os países. O presente trabalho é parte de um projeto bilateral financiado pelo Ministerio de Educación, Cultura y Deporte da Espanha e da Coordenação de Aperfeiçoamento de Pessoal de Nível Superior (CAPES) do Brasil. Desde 2009, o Programa CAPES/DGPU, fruto da parceria entre a CAPES do Brasil e a Direção Geral de Universidades da Espanha, tem apoiado o intercâmbio científico entre instituições de ensino e pesquisa brasileiras e espanholas, visando a consolidação, expansão e internacionalização das instituições de ensino superior e dos centros de pesquisa dos países (COORDENAÇÃO DE APERFEIÇOAMENTO DE PESSOAL DE NÍVEL SUPERIOR, 2015). Laços históricos, culturais, humanos e políticos, além dos acordos e programas de apoio à pesquisa colaborativa internacional firmados desde início da década de 1990, também justificam a escolha da Espanha como parceiro na pesquisa e como foco de análise da colaboração multilateral e bilateral.

Com auxílio de indicadores bibliométricos de produção e colaboração científica, este estudo apresenta uma análise da produção conjunta do Brasil e Espanha na FAE a partir dos artigos publicados em colaboração (multilateral e bilateral) e indexados pela Web of Science (WoS) no período de 1992-2013.

\section{Metodologia}

A coleta dos dados e download dos artigos da produção conjunta do Brasil e Espanha em FAE no período de 1992-2013 foi realizada em dezembro de 2015 no Science Citation Index (SCI) da base multidisciplinar Web of Science. O período de análise compreende o ano do primeiro artigo publicado em colaboração pelos dois países em FAE até 2013, ano que continha um grande número de artigos indexados na WoS no momento da coleta de dados. Na estratégia de busca foi utilizada a seguinte expressão: $\mathrm{CU}=($ Brazil AND Spain $)$ AND WC=(Physics, Particles \& Fields). Além disso, foram recuperados todos os documentos publicados individualmente pelo Brasil e pela Espanha, além dos publicados internacionalmente na área, com o objetivo de analisar a representatividade da produção conjunta dos parceiros envolvidos.

$\mathrm{Na}$ caracterização dos artigos produzidos em colaboração pelos dois países foram utilizados indicadores bibliométricos de produção e colaboração. Indicadores de Produção: ano de publicação Year Published (PY), periódico Source (SO), categoria de assunto Web of Science Category (WC). Indicadores de Colaboração: colaboração multilateral Author 
Address (C1) (publicações de ambos os países junto a terceiros), colaboração bilateral Author Address (C1) (publicações assinadas conjuntamente por autores do Brasil e Espanha). Com relação ao tratamento dos dados, foram utilizados os softwares Bibexcel (análises descritivas), Pajek (visualização da rede de colaboração multilateral e bilateral) e Microsoft Excel 2007 (confecção de tabelas e figuras).

\section{Resultados}

Nesta seção são apresentados e discutidos os resultados da investigação através de indicadores bibliométricos de produção e colaboração científica.

\subsection{Indicadores de Produção}

Inicialmente, foi analisada a contribuição individual do Brasil e Espanha em FAE para a produção mundial indexada na WC Physics, Particles \& Fields da WoS no período de 19922013. Os artigos espanhóis e brasileiros representam 5,57\% e 3,42\% respectivamente do total da investigação, 211.797 documentos produzidos mundialmente em FAE no período. Na Figura 1, observa-se a representatividade dos 20 países mais produtivos em FAE e a posição do Brasil e da Espanha no ranking geral. Nota-se que Estados Unidos $(29,17 \%)$ lidera a lista de nações. Na sequência situam-se Alemanha (15,91\%), Itália (12,43\%), Rússia (10,98\%) e Inglaterra (9,32\%). Em termos de participação, Espanha ocupa o décimo lugar e Brasil o décimo quarto. 


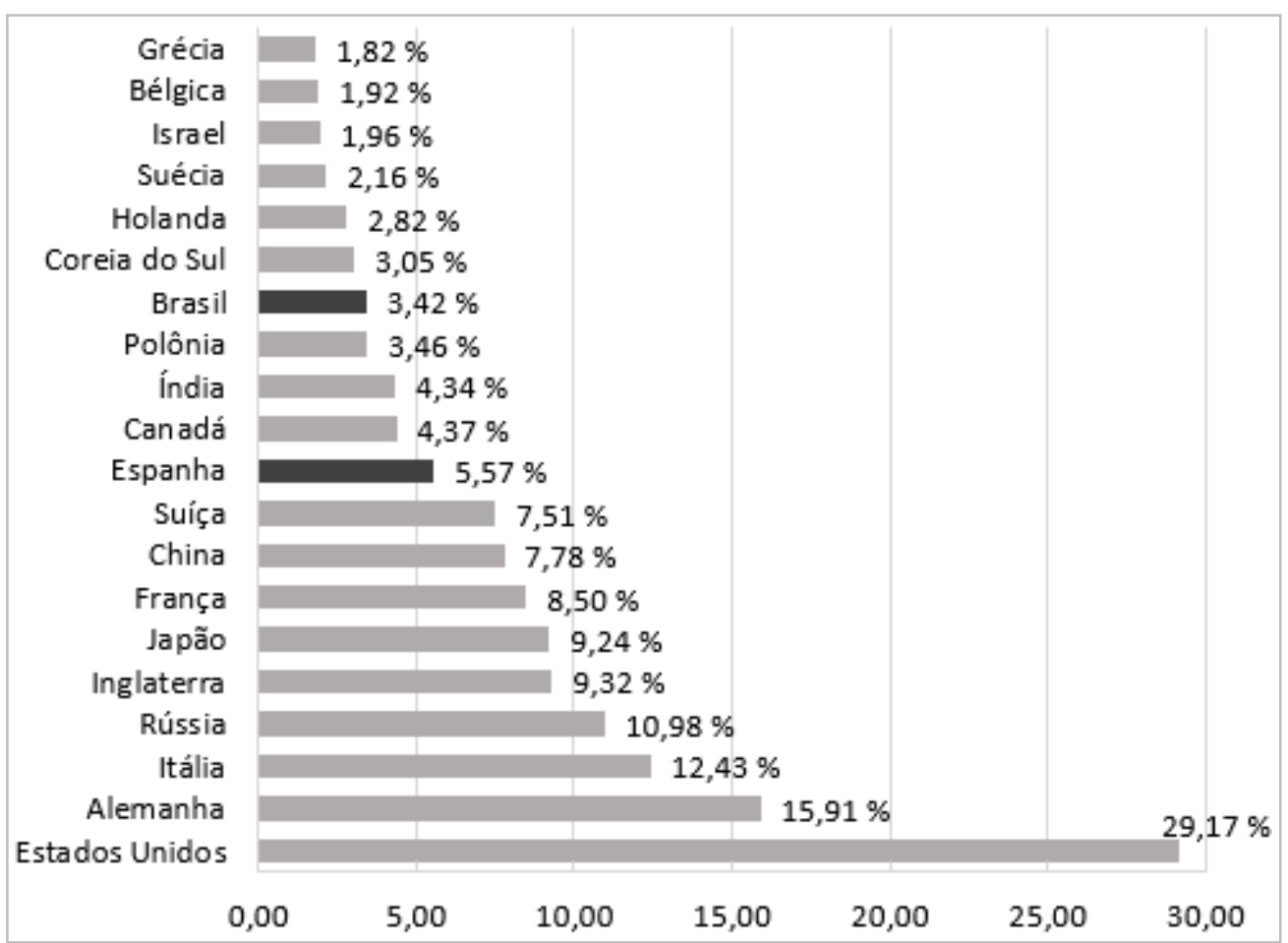

Figura 1 - Países mais produtivos da FAE indexada na WoS, 1992-2013

Fonte: Web of Science

A produção científica da FAE brasileira indexada na WoS entre 1992 e 2013 é constituída por 7.252 artigos, com uma taxa anual de crescimento de 8,7\% para o período analisado (Tabela 1). A criação de novos grupos $\mathrm{CNPq}$, o incremento da participação nas colaborações internacionais e do financiamento e a criação da Rede Nacional de Física de Altas Energias (RENAFAE) em 2008 podem ter sido fatores causais desse crescimento e, consequentemente, da expansão da atividade científica na área (ALVAREZ; VANZ, 2016). Por outro lado, o total de documentos produzidos pela FAE espanhola foi de 11.797 artigos, com uma taxa anual de crescimento de 10,3\% para o período estudado (Tabela 1). Parte desse incremento da produtividade no decorrer dos anos pode estar associado à posição de privilégio que a Espanha detém como Estado-Membro no CERN (EUROPEAN ORGANIZATION FOR NUCLEAR RESEARCH, 2016, documento eletrônico). Bonilla-Calero (2009) aponta que a FAE é uma das seis disciplinas mais produtivas da Física espanhola, sendo a Comunidad Valenciana a que apresenta o maior índice de atividade, seguida pela Comunidad de Madrid, que por sua vez, conta com o maior número de centros de pesquisa produtores, 51 presentes em 19 revistas. 
Tabela 1 - Produção científica da FAE Brasil, Espanha, em colaboração multilateral e bilateral indexada na WoS, 1992-2013

\begin{tabular}{|c|c|c|c|c|c|c|c|c|c|c|}
\hline Ano & $\begin{array}{l}\text { Doc. } \\
\text { Brasil }\end{array}$ & $\begin{array}{l}\mathrm{TC} \\
(\%)\end{array}$ & $\begin{array}{c}\text { Doc. } \\
\text { Espanha }\end{array}$ & $\begin{array}{l}\mathrm{TC} \\
(\%)\end{array}$ & $\begin{array}{c}\text { Doc. } \\
\text { Multilateral }\end{array}$ & $\begin{array}{l}\text { TC } \\
(\%)\end{array}$ & $\begin{array}{c}\text { Doc. } \\
\text { Bilateral }\end{array}$ & $\begin{array}{l}\text { TC } \\
(\%)\end{array}$ & $\begin{array}{c}\text { \% Colab. } \\
\text { BR/ES } \\
\text { para BR }\end{array}$ & $\begin{array}{c}\text { \% Colab. } \\
\text { BR/ES } \\
\text { para ES }\end{array}$ \\
\hline 1992 & 146 & -- & 242 & -- & 11 & -- & 3 & -- & 7,5 & 4,5 \\
\hline 1993 & 140 & $-4,3$ & 281 & 13,9 & 14 & 21,4 & 0 & -- & 10,0 & 5,0 \\
\hline 1994 & 154 & 9,1 & 284 & 1,1 & 18 & 22,2 & 0 & -- & 11,7 & 6,3 \\
\hline 1995 & 176 & 12,5 & 352 & 19,3 & 28 & 35,7 & 0 & -- & 15,9 & 8,0 \\
\hline 1996 & 208 & 15,4 & 399 & 11,8 & 31 & 9,7 & 1 & 100,0 & 14,9 & 7,8 \\
\hline 1997 & 254 & 18,1 & 381 & $-4,7$ & 35 & 11,4 & 3 & 66,7 & 13,8 & 9,2 \\
\hline 1998 & 282 & 9,9 & 466 & 18,2 & 28 & $-25,0$ & 4 & 25,0 & 9,9 & 6,0 \\
\hline 1999 & 309 & 8,7 & 444 & $-5,0$ & 39 & 28,2 & 9 & 55,6 & 12,6 & 8,8 \\
\hline 2000 & 303 & $-2,0$ & 495 & 10,3 & 43 & 9,3 & 4 & $-125,0$ & 14,2 & 8,7 \\
\hline 2001 & 307 & 1,3 & 422 & $-17,3$ & 28 & $-53,6$ & 4 & 0,0 & 9,1 & 6,6 \\
\hline 2002 & 362 & 15,2 & 400 & $-5,5$ & 14 & $-100,0$ & 6 & 33,3 & 3,9 & 3,5 \\
\hline 2003 & 319 & $-13,5$ & 464 & 13,8 & 22 & 36,4 & 5 & $-20,0$ & 6,9 & 4,7 \\
\hline 2004 & 347 & 8,1 & 566 & 18,0 & 27 & 18,5 & 0 & -- & 7,8 & 4,8 \\
\hline 2005 & 311 & $-11,6$ & 551 & $-2,7$ & 17 & $-58,8$ & 2 & 100,0 & 5,5 & 3,1 \\
\hline 2006 & 333 & 6,6 & 539 & $-2,2$ & 20 & 15,0 & 2 & 0,0 & 6,0 & 3,7 \\
\hline 2007 & 393 & 15,3 & 608 & 11,3 & 17 & $-17,6$ & 5 & 60,0 & 4,3 & 2,8 \\
\hline 2008 & 331 & $-18,7$ & 591 & $-2,9$ & 15 & $-13,3$ & 1 & $-400,0$ & 4,5 & 2,5 \\
\hline 2009 & 326 & $-1,5$ & 674 & 12,3 & 22 & 31,8 & 3 & 66,7 & 6,7 & 3,3 \\
\hline 2010 & 362 & 9,9 & 692 & 2,6 & 46 & 52,2 & 3 & 0,0 & 12,7 & 6,6 \\
\hline 2011 & 547 & 33,8 & 848 & 18,4 & 151 & 69,5 & 6 & 50,0 & 27,6 & 17,8 \\
\hline 2012 & 646 & 15,3 & 1.064 & 20,3 & 300 & 49,7 & 2 & $-200,0$ & 46,4 & 28,2 \\
\hline 2013 & 696 & 7,2 & 1.034 & $-2,9$ & 288 & $-4,2$ & 9 & 77,8 & 41,4 & 27,9 \\
\hline Total & 7.252 & 8,7 & 11.797 & 10,3 & 1.214 & 15,0 & 72 & -- & 16,7 & 10,3 \\
\hline
\end{tabular}

Fonte: Web of Science

Nota: TC - Taxa de crescimento

As publicações em colaboração multilateral (entre Brasil, Espanha e outros países) totalizaram 1.214 artigos no período, representando $16,7 \%$ da produção científica brasileira e 10,3\% da espanhola, isto é, a colaboração científica em FAE é mais representativa para o Brasil do que para a Espanha no período analisado. Esse indicador confirma os resultados encontrados por Moura et al. (2015) em análise da produção conjunta (Brasil/Espanha) para todas as áreas do conhecimento. Dentre esse conjunto de publicações, 5,9\% (72 documentos) são de colaboração bilateral, ou seja, artigos assinados somente por Brasil e Espanha. A carência de informações referentes a artigos publicados em alguns anos, principalmente no início do período, impossibilitou o cálculo da taxa anual de crescimento para a colaboração bilateral.

A taxa anual de crescimento da colaboração para ambos os países $(15,0 \%)$ é superior ao crescimento individual da produção científica de cada um deles. No caso do Brasil, o papel 
articulador da RENAFAE para as colaborações brasileiras nos programas internacionais, como os experimentos realizados no CERN (Suíça) e Observatório Pierre Auger (Argentina) é justificado pela intensificação do aumento da produção a partir de 2008 (CENTRO BRASILEIRO DE PESQUISAS FÍSICAS, 2011). Com relação à Espanha, Bonilla-Calero (2009) comenta que a FAE apresenta o índice de colaboração internacional mais alto dentro as subáreas da Física espanhola, atrás apenas da Astronomia e Astrofísica.

Dentre os 1.214 artigos publicados por Brasil e Espanha, observa-se 72 deles (5,9\%) em colaboração bilateral, com alta variabilidade quanto ao número de publicações anuais, representando uma cooperação eventual de curto prazo (Tabela 1). A forte prevalência da colaboração multilateral $(94,1 \%)$ é justificada pela participação de ambos os países nos grandes consórcios multinacionais dos laboratórios CERN (Suíça) e Fermilab (Estados Unidos). Notase um início recente das atividades de pesquisa entre os dois países visto que os primeiros artigos em colaboração foram publicados em 1992. Desde alguns anos, o Programa CAPES/DGPU, fruto da parceria entre a Coordenação de Aperfeiçoamento de Pessoal de Nível Superior do Brasil e a Direção Geral de Universidades da Espanha, tem apoiado os projetos conjuntos de pesquisa com vistas ao intercâmbio científico entre instituições de ensino superior brasileiras e espanholas (COORDENAÇÃO DE APERFEIÇOAMENTO DE PESSOAL DE NÍVEL SUPERIOR, 2015).

Com o objetivo de aprofundar as análises referentes aos padrões de produção e divulgação de resultados científicos em FAE de cada um dos países envolvidos, foram identificados os tipos de periódico de publicação, relacionando-os com sua procedência e quartil. Os indicadores mostram que tanto o Brasil quanto a Espanha têm perfis similares de publicação (Tabela 2). A análise do quartil das revistas utilizadas para veiculação das descobertas científicas indica a paridade do número de publicações em revistas Q1, para Brasil $(89,1 \%)$ e Espanha $(85,7 \%)$. A totalidade das publicações de ambos os países está concentrada em veículos internacionais de língua inglesa. O estudo realizado mostra que a colaboração multilateral favorece a publicação em revistas Q1 tendo em vista que 90,6\% dos artigos multilaterais foram publicados em periódicos desse quartil, enquanto nas publicações bilaterais o percentual cai para $80,6 \%$. Esse indicador demonstra que tanto para o Brasil quanto para a Espanha a colaboração bilateral em FAE não traz benefícios suficientes em termos de impacto. Quando analisada a representatividade do número de publicações em revistas Q1 da 
colaboração multilateral na produção científica da cada país, observa-se que o peso das publicações nesse tipo de revista é maior para o Brasil (17,0\%) do que para a Espanha (10,9\%).

Tabela 2 - Documentos da FAE Brasil, Espanha, em colaboração multilateral e bilateral indexada na WoS por tipo de revista, 1992-2013

\begin{tabular}{lcccccccc}
\hline $\begin{array}{c}\text { Tipo de } \\
\text { revista }\end{array}$ & $\begin{array}{c}\text { Doc. } \\
\text { Brasil }\end{array}$ & \% & $\begin{array}{c}\text { Doc. } \\
\text { Espanha }\end{array}$ & \% & $\begin{array}{c}\text { Doc. } \\
\text { Multilateral }\end{array}$ & $\%$ & $\begin{array}{c}\text { Doc. } \\
\text { Bilateral }\end{array}$ & $\%$ \\
\hline Revistas Q1 & 6.460 & 89,1 & 10.113 & 85,7 & 1.100 & 90,6 & 58 & 80,6 \\
Revistas Q2 & 709 & 9,8 & 1.492 & 12,6 & 111 & 9,1 & 13 & 18,1 \\
Revistas Q3 & 72 & 1,0 & 137 & 1,2 & 1 & 0,1 & 1 & 1,4 \\
Revistas Q4 & 4 & 0,1 & 29 & 0,2 & 0 & 0,0 & 0 & 0,0 \\
Total & $\mathbf{7 . 2 5 2}$ & $\mathbf{1 0 0 , 0}$ & $\mathbf{1 1 . 7 9 7}$ & $\mathbf{1 0 0 , 0}$ & $\mathbf{1 . 2 1 4}$ & $\mathbf{1 0 0 , 0}$ & $\mathbf{7 2}$ & $\mathbf{1 0 0 , 0}$ \\
\hline
\end{tabular}

Fonte: Web of Science

Nota: Os dados sobre o Quartil das revistas de publicação foram coletados do Journal Citation Reports (JCR) 2014.

Os resultados apresentados na Tabela 2 demonstram a alta qualidade das revistas utilizadas para veicular os resultados da pesquisa brasileira e espanhola em FAE. A análise da colaboração entre Brasil e Espanha em todas as áreas do conhecimento apresenta um panorama diferente, tendo em vista que apenas 41,97\% dos artigos publicados por ambos os países estão em revistas Q1, um percentual bastante reduzido em relação ao da produção científica dos dois países em FAE (80,6\%). Ao analisar somente a produção científica brasileira em todas as áreas, $30,98 \%$ dos documentos estão publicados em revistas Q1. Este percentual sobe para 49,05\% no caso das publicações da Espanha no período 2006-2012 (VANZ et al., 2016). A análise de todas as áreas, portanto, permite concluir que a colaboração bilateral é proveitosa para o Brasil, tendo em vista que o percentual de publicação em revistas Q1 é superior ao atingido por publicações sem colaboração. A colaboração multilateral, no entanto, favorece a publicação em revistas Q1 tanto na FAE quanto na análise da ciência em todas as áreas do conhecimento.

Na análise da produção científica em FAE não foram identificadas revistas nacionais, aquelas publicadas pelo Brasil ou pela Espanha. A área possui um conjunto relativamente pequeno de revistas publicadas em língua inglesa, altamente especializadas e com alto desempenho no que diz respeito às citações e consequentemente, a maioria situa-se no primeiro Quartil. Como a área concentra sua produção científica nesses periódicos, a análise de Quartil apresenta bons resultados individuais para Brasil e Espanha. Em termos de produtividade, destacaram-se algumas das revistas com maior tradição de publicação de resultados científicos em FAE, sendo elas: Physical Review D, 29 artigos (40,3\%), Physics Letters B, 10 artigos 
(13,9\%), Journal of High Energy Physics, 3 artigos (4,2\%), European Physical Journal C, 2 $\operatorname{artigos}(2,8 \%)$.

A análise das publicações científicas indexadas na WoS do Brasil e Espanha em FAE por temática foi realizada por meio do campo WC e teve como finalidade a classificação dos registros bibliográficos coletados pelo assunto dos periódicos utilizados. Quanto ao perfil de publicação por temática, observa-se paridade do percentual de publicações da categoria Astronomy \& Astrophysics, para Brasil (48,0\%) e para Espanha (47,0\%) (Tabela 3). Vislumbrase uma leve superioridade brasileira na WC Physics, Nuclear $(28,4 \%)$. A participação da Astronomia \& Astrofísica e da Física Nuclear nas publicações da FAE brasileira e espanhola decorre não somente da significativa influência de ambas no contexto histórico, mas também do desenvolvimento da pesquisa no âmbito nacional e internacional. Considerada em princípio como parte da Física Nuclear, a FAE é um dos campos mais importantes de investigação básica das ciências físicas (COLLAZO-REYES; LUNA-MORALES, 2002). Nota-se que a Espanha teve maior desempenho individual do que o Brasil nas categorias relacionadas com áreas associadas aos métodos de detecção e controle de reações nucleares (Nuclear Science \& Technology), técnicas (Spectroscopy) e instrumentais vinculadas a detectores e aceleradores de partículas (Instruments \& Instrumentation) (CHAVES; SHELLARD, 2005).

Tabela 3 - Distribuição da produção científica da FAE Brasil e Espanha indexada na WoS por categoria WC, 1992-2013

\begin{tabular}{lcccccccc}
\hline \multicolumn{1}{c}{$\begin{array}{c}\text { Categoria } \\
\text { WC }\end{array}$} & $\begin{array}{c}\text { Doc. } \\
\text { Brasil }\end{array}$ & $\boldsymbol{\%}$ & $\begin{array}{c}\text { Doc. } \\
\text { Espanha }\end{array}$ & $\boldsymbol{\%}$ & $\begin{array}{c}\text { Doc. } \\
\text { Multilateral }\end{array}$ & \% & $\begin{array}{c}\text { Doc. } \\
\text { Bilateral }\end{array}$ & \% \\
\hline Astron. \& Astrophysics & 4.188 & 48,0 & 6.337 & 47,0 & 679 & 56,5 & 50 & 67,6 \\
Physics, Nuclear & 2.481 & 28,4 & 3.256 & 24,2 & 413 & 34,4 & 17 & 23,0 \\
Physics, Multidisciplinary & 490 & 5,6 & 602 & 4,5 & 12 & 1,0 & 4 & 5,4 \\
Physics, Mathematical & 382 & 4,4 & 260 & 1,9 & 1 & 0,1 & 0 & 0,0 \\
Nuclear Sci \& Technology & 378 & 4,3 & 988 & 7,3 & 32 & 2,7 & 1 & 1,4 \\
Spectroscopy & 371 & 4,2 & 974 & 7,2 & 32 & 2,7 & 1 & 1,4 \\
Instrum \& Instrumentation & 371 & 4,2 & 974 & 7,2 & 32 & 2,7 & 1 & 1,4 \\
Comp Sci, Theory \& Methods & 42 & 0,5 & 60 & 0,4 & 0 & 0,0 & 0 & 0,0 \\
Meteor \& Atmospheric Sci. & 7 & 0,1 & 0 & 0,0 & 0 & 0,0 & 0 & 0,0 \\
Geosciences, Multidiscip. & 7 & 0,1 & 0 & 0,0 & 0 & 0,0 & 0 & 0,0 \\
Engineering, Aerospace & 7 & 0,1 & 0 & 0,0 & 0 & 0,0 & 0 & 0,0 \\
Physics, Fluids \& Plasmas & 6 & 0,1 & 14 & 0,1 & 0 & 0,0 & 0 & 0,0 \\
Materials Sci, Multidiscip. & 1 & 0,0 & 8 & 0,1 & 0 & 0,0 & 0 & 0,0 \\
Physics, Applied & 1 & 0,0 & 0 & 0,0 & 0 & 0,0 & 0 & 0,0 \\
Physics, Cond Matter & 1 & 0,0 & 8 & 0,1 & 0 & 0,0 & 0 & 0,0 \\
Total & $\mathbf{8 . 7 3 3}$ & $\mathbf{1 0 0 , 0}$ & $\mathbf{1 3 . 4 8 1}$ & $\mathbf{1 0 0 , 0}$ & $\mathbf{1 . 2 0 1}$ & $\mathbf{1 0 0 , 0}$ & $\mathbf{7 4}$ & $\mathbf{1 0 0 , 0}$ \\
\hline Font & & & & & & &
\end{tabular}

Fonte: Web of Science 
Nota: a soma das publicações excede o total real da produção científica visto que há revistas atribuídas a mais de uma categoria WC da WoS.

Ao analisar a especialização temática da produção bilateral (Brasil e Espanha), observase que a área de maior destaque é a Astronomy \& Astrophysics, com quase $70 \%$ do total das publicações. Embora prevaleça a colaboração em grandes redes de países nas disciplinas consideradas Big Science (Astronomia, Física de Partículas, Física Nuclear), percebe-se também a importância e a necessidade das colaborações bilaterais em campos experimentais para compartilhamento de equipamentos, instalações, obtenção e/ou ampliação de financiamento e recursos a partir de acordos e convênios de cooperação.

\subsection{Indicadores de Colaboração}

Em análise das 1.214 publicações que compõem o corpus da produção multilateral (Brasil, Espanha e outros países), verificou-se que existem artigos assinados por até 43 países. Conforme foi apontado anteriormente a colaboração bilateral representa 5,9\% da totalidade das publicações, enquanto a colaboração trilateral 5,8\% (Brasil, Espanha e outro país) e os documentos assinados por mais de 20 países alcançam $62,8 \%$.

A FAE brasileira apresenta uma taxa de colaboração de $53,2 \%$, ou seja, pouco mais da metade da sua produção científica tem sido assinada com instituições de outras nações. Durante o período observado, o Brasil publicou artigos em periódicos internacionais com autores de 85 países diferentes, destacando-se as colaborações com Estados Unidos (27,3\%), Rússia (20,8\%), França (20,2\%), Alemanha (20,1\%) e Reino Unido (19,6\%). Já a FAE espanhola apresenta uma taxa de colaboração amplamente superior à do Brasil, 73,4\% dos artigos espanhóis foram assinados por mais de um país, com destaque para as colaborações com Estados Unidos (32,6\%), Itália (32,3\%), Alemanha (31,4\%), Reino Unido (25,9\%) e França (25,6\%). A taxa da colaboração internacional da FAE espanhola se aproxima aos $71 \%$ registrados por BonillaCalero (2009) em análise da colaboração da Espanha nos artigos indexados na WoS no período de 2000-2005.

O alto investimento de dinheiro necessário para a realização da pesquisa experimental em FAE em redes de colaboração internacional pode justificar a prevalência de artigos teóricos em países em desenvolvimento como é o caso do Brasil. Um estudo realizado pela Sociedade Brasileira de Física em 2012 mostrou que, dentre o corpo docente da FAE, a parcela de 
professores da subárea experimental $(13,1 \%)$ é notoriamente inferior com relação à parcela da subárea teórica $(37,5 \%)$. Apesar da comunidade experimental se organizar por meio da RENAFAE, falta ainda uma maior conscientização sobre o desenvolvimento de instrumentação especializada e laboratórios nacionais que possibilitem a ampliação do ensino e da pesquisa (SOCIEDADE BRASILEIRA DE FÍSICA, 2012).

Ao examinar os países colaboradores que assinaram artigos conjuntamente com Brasil e Espanha entre 1992 e 2013, observa-se também que Estados Unidos (85,3\%) é o principal parceiro de ambos os países (Tabela 4). A continuação, situam-se França (84,1\%), Alemanha $(83,7 \%)$, Reino Unido (83,5\%) e Rússia (80,5\%). Percebe-se como a colaboração multilateral, com presença do Brasil e Espanha conjunta nas publicações, intensifica as parcerias com aqueles países líderes no ranking de produtividade.

Tabela 4 - Colaboradores mais frequentes em FAE do Brasil, Espanha e colaboração multilateral, 1992-2013

\begin{tabular}{lcccccc}
\hline \multicolumn{1}{c}{$\begin{array}{c}\text { País } \\
\text { colaborador }\end{array}$} & $\begin{array}{c}\text { Doc. } \\
\text { Brasil }\end{array}$ & $\boldsymbol{\%}$ & $\begin{array}{c}\text { Doc. } \\
\text { Espanha }\end{array}$ & $\boldsymbol{\%}$ & $\begin{array}{c}\text { Doc. } \\
\text { Multilateral }\end{array}$ & \% \\
\hline Alemanha & 1.458 & 20,1 & 3.708 & 31,4 & 1.016 & 83,7 \\
Áustria & 754 & 10,4 & 1.155 & 9,8 & 736 & 60,6 \\
Brasil & $\mathbf{7 . 2 5 2}$ & $\mathbf{1 0 0 , 0}$ & $\mathbf{1 . 2 1 4}$ & $\mathbf{1 0 , 2}$ & $\mathbf{1 . 2 1 4}$ & $\mathbf{1 0 0 , 0}$ \\
China & 1.006 & 13,9 & 1.474 & 12,5 & 682 & 56,2 \\
Colômbia & 685 & 9,4 & 548 & 4,6 & 511 & 42,1 \\
Espanha & $\mathbf{1 . 2 1 4}$ & $\mathbf{1 6 , 7}$ & $\mathbf{1 1 . 7 9 7}$ & $\mathbf{1 0 0 , 0}$ & $\mathbf{1 . 2 1 4}$ & $\mathbf{1 0 0 , 0}$ \\
Estados Unidos & 1.977 & 27,3 & 3.848 & 32,6 & 1.036 & 85,3 \\
França & 1.463 & 20,2 & 3.024 & 25,6 & 1.021 & 84,1 \\
Grécia & 811 & 11,2 & 1.413 & 12 & 775 & 63,8 \\
Holanda & 984 & 13,6 & 1.920 & 16,3 & 779 & 64,2 \\
Índia & 643 & 8,9 & 972 & 8,2 & 362 & 29,8 \\
Itália & 1.384 & 19,1 & 3.813 & 32,3 & 962 & 79,2 \\
México & 726 & 10,0 & 601 & 5,1 & 433 & 35,7 \\
Polônia & 1.066 & 14,7 & 1.576 & 13,4 & 922 & 75,9 \\
Portugal & 808 & 11,1 & 997 & 8,5 & 678 & 55,8 \\
Reino Unido & 1.425 & 19,6 & 3.051 & 25,9 & 1.014 & 83,5 \\
Rep. Tcheca & 1.020 & 14,1 & 996 & 8,4 & 841 & 69,3 \\
Rússia & 1.512 & 20,8 & 2.766 & 23,4 & 977 & 80,5 \\
Suécia & 803 & 11,1 & 901 & 7,6 & 654 & 53,9 \\
Suíça & 1.053 & 14,5 & 2.731 & 23,1 & 911 & 75,0 \\
\hline Fonte: Web of Science & & & & & &
\end{tabular}


Os principais países colaboradores do Brasil em FAE são basicamente os mesmos que foram identificados por Vanz (2009), Adams e King (2009) e Fundação de Amparo à Pesquisa do Estado de São Paulo (2011) nas coautorias da ciência brasileira. O predomínio das relações de cooperação com os parceiros citados pode ser explicado pelos acordos e programas de apoio à pesquisa colaborativa internacional que são firmados pelo Brasil. Já no caso da FAE espanhola, os principais colaboradores (Estados Unidos, Alemanha e Itália) são os mesmos apontados por Bonilla-Calero (2009) quando analisou os principais parceiros da Espanha na Física de Partículas na grande rede internacional entre 2000 e 2005. Na Figura 2, apresenta-se o grafo correspondente à rede de colaboração dos países com maior número de publicações em coautoria (até 442 artigos) com Brasil/Espanha no período de 1992-2013.

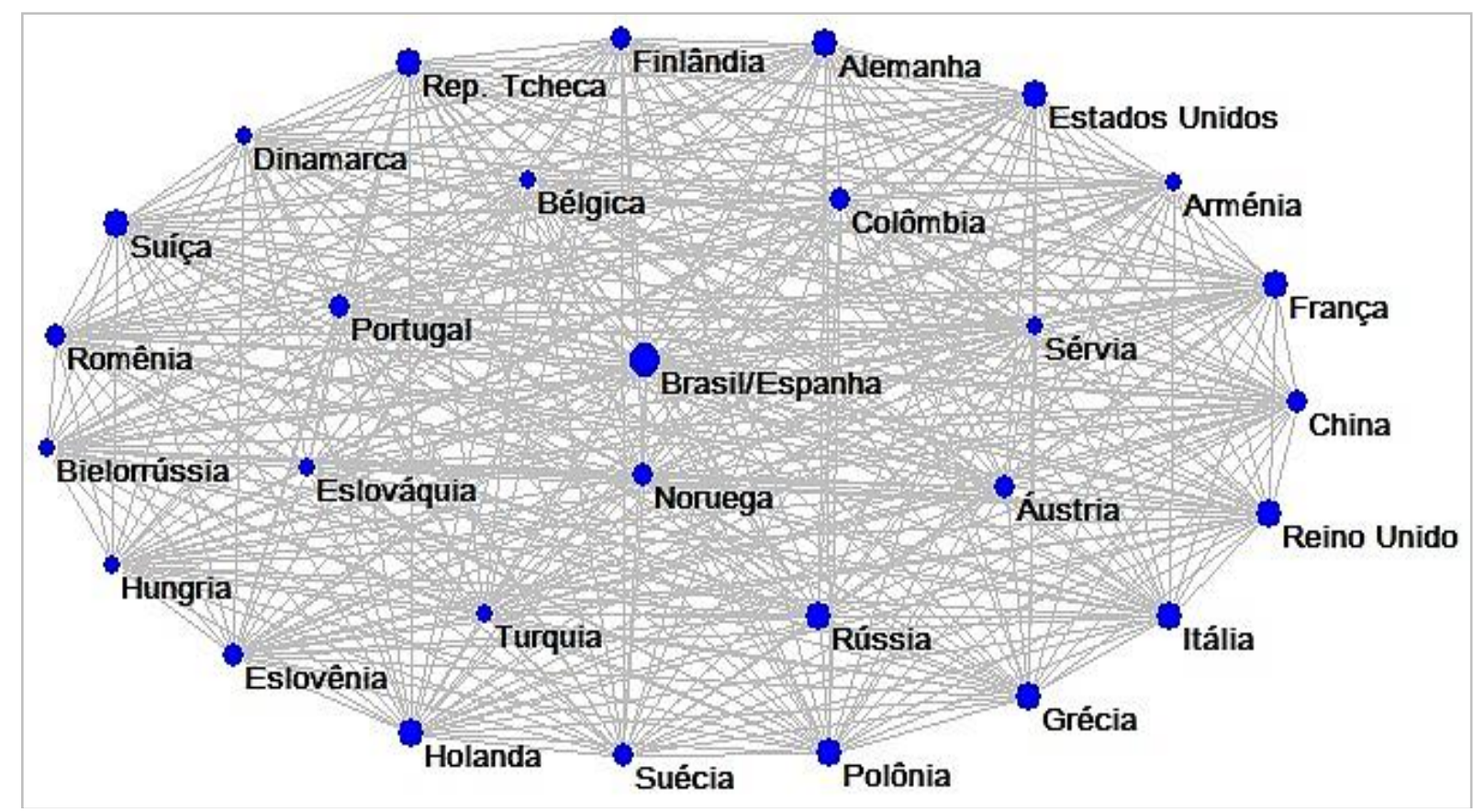

Figura 2 - Colaboração Brasil/Espanha com outros países, 1992-2013 Fonte: Web of Science

A rede de colaboração multilateral (Brasil/Espanha e outros países) mostra-se compacta. O nível de agrupamento na FAE revela a alta centralização da investigação científica em um número relativamente baixo de países, com presença das nações com maior tradição e produtividade na área: Estados Unidos (América do Norte), França, Itália, Alemanha, Rússia e Reino Unido (Europa).

Todos os 72 artigos em colaboração bilateral (Brasil/Espanha) pertencem à subárea teórica da FAE e são vinculados a pequenos grupos com no máximo cinco pesquisadores filiados a instituições das regiões Sudeste e Nordeste do Brasil e a centros mistos da Espanha 
como o Consejo Superior de Investigaciones Científicas (CSIC) e a Universidad de Valencia (UV) (Figura 3). O Consejo Superior de Investigaciones Científicas (CSIC) é a maior instituição pública espanhola dedicada à pesquisa e desempenha um papel fundamental na política de C\&T do país, pois abarca desde a pesquisa básica até a transferência do conhecimento ao setor produtivo. O CSIC emprega 6\% dos pesquisadores espanhóis e é responsável por cerca de $20 \%$ da produção científica nacional (CONSEJO SUPERIOR DE INVESTIGACIONES CIENTÍFICAS, 2016).

Quanto aos focos temáticos de pesquisa em diferentes subdomínios da produção bilateral, destacou-se o cluster conformado pelas palavras-chave: 'Universe', 'Cosmological Constant', 'Dark Energy', 'Supernovae', 'Constraint' e 'Lambda', referindo-se aos artigos do campo da Cosmologia, especificamente aos elementos do Modelo Padrão Cosmológico ou Lambda-Cold Dark Matter ( $\Lambda$ CDM). As instituições paulistas (USP, UNESP, UNICAMP) e valencianas (UV) mais produtivas no período de 1992-2013, encontram-se representadas neste agrupamento.

A maior centralidade na rede da UV/CSIC (Figura 3) é ressaltada pelo trabalho conjunto desenvolvido pelo Instituto de Física Corpuscular, dedicado à investigação em Física Nuclear e de Partículas, desde a década de 1980 e pela província de Valencia, região com maior tradição nesses campos científicos. O programa de investigação inaugurado em 1950 pelo físico espanhol Joaquín Catalá de Alemany, em Valencia, deu origem institucional à pesquisa experimental em Física Nuclear e de Partículas em Espanha. O grupo de Valencia fomentou uma tradição que continua ativa até hoje (CEBA; NAVARRO; VELASCO, 2011). Dentre as instituições brasileiras as universidades paulistas USP, UNESP e UNICAMP apresentaram o maior grau de conectividade com a UV/CSIC, ressaltando a excelência e tradição dos seus programas vinculados a temáticas dentro das linhas de pesquisas experimentais (Exp. de Altas Energias, Fenomenologia de Partículas, Astrofísica de Neutrinos, Raios Cósmicos, Partículas Elementares e Campos). No conjunto de artigos da colaboração bilateral (Brasil-Espanha), 50 documentos $(69,4 \%)$ envolvem apenas uma universidade brasileira e uma universidade espanhola, inferindo-se que os vínculos institucionais em FAE entre os dois países acontecem a partir de projetos/convênios específicos de cooperação. A continuação, destacaram-se os artigos com três instituições $(19,4 \%)$, quatro instituições $(9,7 \%)$ e cinco instituições $(1,4 \%)$. O perfil de colaboração entre estes dois países na FAE mostra-se bastante diferente de outras áreas. Na Medicina, por exemplo, 15,88\% dos artigos indexados na Scopus no período 2002- 
2011 foram assinados por duas instituições (uma brasileira e outra espanhola), 18,91\% assinados por três instituições e 13,78\% assinados por quatro instituições (ARROYO et al., 2016).

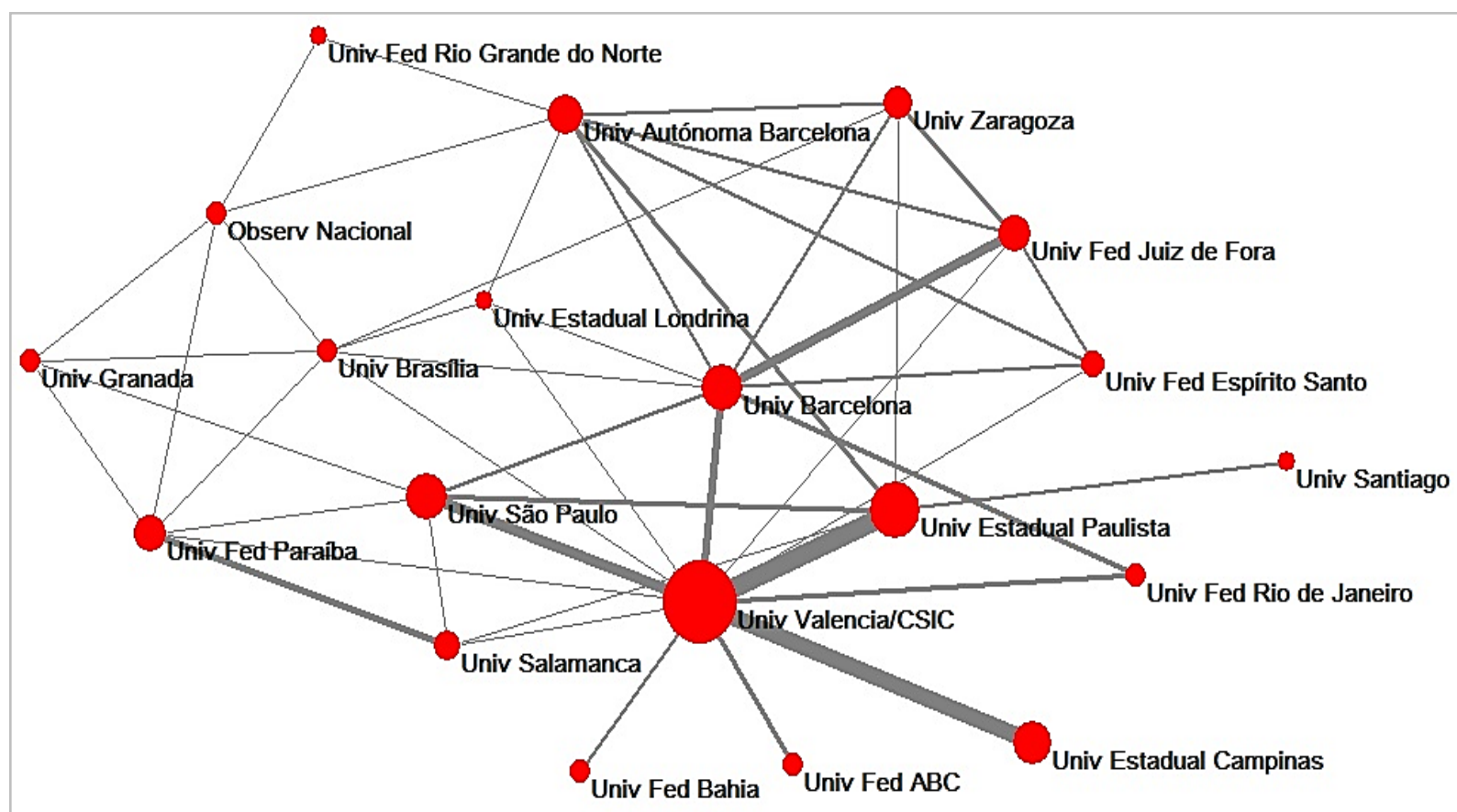

Figura 3 - Principais instituições da colaboração bilateral (Brasil-Espanha) em FAE, 1992-2013 Fonte: Web of Science

Em concordância com Bonilla-Calero (2009), instituições de Catalunha e Valencia foram as que apresentaram as maiores taxas de colaboração. Em análise qualitativa dos artigos da colaboração bilateral, buscou-se a indicação de financiamento ou agradecimento às agências financiadoras. Observou-se a presença de fundos de apoio à pesquisa vindos de subsídios de colaboração Europa-América Latina em 21 artigos da totalidade da produção conjunta, como por exemplo: colaboração UV/USP e colaboração UV/UNICAMP (Ministerio de Economía y Competitividad/FAPESP/CNPq); colaboração UV/UFPB (CSIC/CNPq); colaboração UAB/ON/UFRN (Ministerio de Economía y Competitividad/CAPES/CNPq); colaboração UAB/UFES e colaboração UV/UFABC (Ministerio de Ciencia e Innovación/CNPq); colaboração UNIOVI/UFPB (Fundación para el Fomento en Asturias de la Investigación Científica Aplicada y la Tecnología/CAPES/CNPq); colaboração UAB/UEL (Ministerio de Educación y Ciencia/CNPq); colaboração UAB/UFES (Recerca de la Generalitat de Catalunya/CNPq/CAPES); colaboração UB/UFJF (Recerca de la Generalitat de Catalunya/CNPq/FAPEMIG); colaboração UV/USAL/UNESP (Ministerio de Educación y 
Ciencia/Centro Nacional de Física de Partículas, Astropartículas y Nuclear/CNPq/FAPESP). Destaca-se que grandes universidades da Espanha como a Universidad Autónoma de Madrid e a Universidad Complutense de Madrid não aparecem entre as principais instituições colaboradoras da Figura 3.

\section{Considerações finais}

Ainda que nem todas as atividades de cooperação científica deem lugar a uma publicação conjunta, a coautoria é um dos resultados das interações entre pesquisadores, e um dos instrumentos mais importantes para mensurar as relações entre autores, instituições e países.

A análise da produção conjunta entre Brasil e Espanha em FAE por indicadores bibliométricos de produção e colaboração revelou que a taxa de crescimento da colaboração multilateral (Brasil, Espanha e outros países) é superior ao crescimento individual da produção científica de cada um deles. No caso do Brasil, o papel articulador da RENAFAE possibilitou o aumento do número de acordos e iniciativas que tivessem como finalidade o incremento da cooperação e intercâmbio científico entre pesquisadores filiados a instituições brasileiras e estrangeiras atuantes na área. Da mesma maneira, se destaca a agência estatal Consejo Superior de Investigaciones Científicas (CSIC) na promoção e coordenação da participação espanhola nos grandes experimentos do CERN.

Os indicadores demonstram que a colaboração internacional (multilateral) favorece a publicação em revistas Q1 e o incremento do número de citações. A queda do percentual das publicações bilaterais em revistas Q1 aponta que tanto para o Brasil quanto para a Espanha, a colaboração bilateral em FAE não traz benefícios suficientes em termos de impacto. A respeito da especialização temática da produção conjunta (Brasil e Espanha), a prevalência dos artigos bilaterais na categoria Astronomy \& Astrophysics ressalta a necessidade e a importância das colaborações desse tipo em áreas experimentais da Big Science a partir da constituição de acordos e convênios de cooperação.

Na FAE, a colaboração internacional é fundamental, pois melhora os indicadores quanto maior for o número de países envolvidos. Verificou-se que a colaboração multilateral intensifica os vínculos com aqueles países líderes no ranking de produtividade. No entanto, a participação de Brasil e Espanha nas redes multinacionais não implica uma marcada especialização. A integração nesses grandes grupos é produto das características próprias da 
área e da necessidade dos países de complementar capacidades e infraestrutura. A colaboração bilateral entre Brasil e Espanha teve início recente, no ano de 1992, e se concentra em artigos da FAE teórica. A continuidade da pesquisa permitirá aprofundar os efeitos do impacto da produção conjunta dos países envolvidos em diferentes níveis: colaboração multilateral e colaboração bilateral.

\section{Referências}

ADAMS, J.; KING, C. Global research report: Brazil. Research and collaboration in the new geography of science. Leeds: Thomson Reuters, 2009.

ALVAREZ, G. R.; VANZ, S. A. S. O desenvolvimento da Física de altas energias no Brasil: produção científica, programas de pós-graduação e grupos de pesquisa. RBPG: Revista Brasileira de Pós-graduação, Brasília, v. 13, n. 31, p. 433-460, 2016.

ARROYO, A. A. et al. Un análisis bibliométrico en el area de la Medicina: colaboración científica entre Brasil y España (2002-2011). Investigación Bibliotecológica, Ciudad de México, v. 69, n. 2, p. 205-229, 2016.

BONILLA-CALERO, A. I. La colaboración y la visibilidad en las disciplinas de Física en Science Citation Index y arXiv (2000-2005). 2009. $476 \mathrm{f}$. Tese (Doutorado) - Universidad Carlos III de Madrid, Departamento de Biblioteconomía y Documentación, Getafe, 2009.

BORDONS, M.; GÓMEZ, I. Collaboration networked in science. In: CRONIN, B.; ATKINS, H. B. (Ed.). The web of knowledge: a festschrift in honor of Eugene Garfield. New Jersey: ASIS, 2000. p. 197-214.

BRAUN, T. et al. International co-authorship patterns in physics and its subfields, 1981-1985. Scientometrics, Amsterdam, v. 24. n. 2, 181-200, 1992.

CEBA, A.; NAVARRO, V.; VELASCO, J. Los orígenes de la investigación experimental en Física Nuclear y de Partículas en Espanha. Revista Española de Física, Madrid, v. 25, n. 2, p. 54-61, 2011.

\section{CENTRO BRASILEIRO DE PESQUISAS FÍSICAS - CBPF. Plano Diretor do CBPF}

2011-2015. Rio de Janeiro: CBPF, 2011. Disponível em:

<http://portal.cbpf.br/attachments/o_cbpf/pdfs/PlanoDiretor.pdf>. Acesso em: 23 nov. 2014.

CHAVES, A.; SHELLARD, R. C. Física para o Brasil: pensando o futuro. São Paulo: SBF, 2005. Disponível em: <http://www.sbfisica.org.br/v1/arquivos_diversos/ publicacoes/FisicaBrasil_Dez05.pdf>. Acesso em: 23 mar. 2015.

CHAVES, A. et al. Relatório apresentado ao Ministério da Ciência e Tecnologia sobre alguns aspectos da Física brasileira. 2002. Disponível em:

<http://www.sbfisica.org.br/arquivos/RelatorioMCT.pdf>. Acesso em: 22 nov. 2014. 
COLLAZO-REYES, F.; LUNA-MORALES, M. E. Física mexicana de partículas elementales: organización, producción científica y crecimiento. Interciencia, Santiago, v. 27, n. 7, p. 347-353, 2002.

CONSEJO SUPERIOR DE INVESTIGACIONES CIENTÍFICAS. Presentación. Madrid: CSIC, 2016. Disponível em: <http://www.csic.es/presentacion>. Acesso em: 03 nov. 2016.

COORDENAÇÃO DE APERFEIÇOAMENTO DE PESSOAL DE NÍVEL SUPERIOR. Programa CAPES-DGPU. Brasília, DF: CAPES, 2015. Disponível em:

<http://www.capes.gov.br/cooperacaointernacional/espanha/capesdgpu>. Acesso em: 31 mar. 2016.

DE FILIPPO, D.; BARRERE, R.; GÓMEZ, I. Características e impacto de la producción científica en colaboración entre Argentina y España. Revista Iberoamericana de Ciencia, Tecnología y Sociedad, Buenos Aires, v. 6, n. 16, p. 179-200, 2010.

DUARTE, R. P. Cooperação Internacional para o Desenvolvimento em Ciência e Tecnologia: a participação brasileira na Organização Europeia para Pesquisa Nuclear (CERN). Journal of Technology Management \& Innovation, Santiago, v. 3, n. 4, p. 133-151, 2008.

EUROPEAN ORGANIZATION FOR NUCLEAR RESEARCH - CERN. Member States. Geneva: CERN, 2016. Disponível em: <https://home.cern/about/member-states>. Acesso em: 21 jul. 2016.

FUNDAÇÃO DE AMPARO À PESQUISA DO ESTADO DE SÃO PAULO - FAPESP. Análise da produção científica a partir de indicadores bibliométricos. In: Indicadores de ciência, tecnologia e inovação em São Paulo 2010. São Paulo: FAPESP, 2011. cap. 4.

GLÄNZEL, W.; SCHUBERT, A. Analyzing scientific networks through coauthorship. In: MOED, H. F.; GLÄNZEL, W.; SCHMOCH, U. Handbook of quantitative science and technology research. Netherlands: Kluwer Academic, 2004. p. 257-276.

KATZ, J. S.; MARTIN, B. R. What is research collaboration? Research Policy, Amsterdam, n. 26, p. 1-18, 1997.

LEFEVRE, C. Technology transfer @ CERN: from research to everyday life. Geneva: CERN, 2007. Disponível em: <http://cds.cern.ch/record/1106357>. Acesso em: 23 fev. 2016.

LETA, J.; CRUZ, C. H. B. A produção científica brasileira. In: VIOTTI, E. B.; MACEDO, M. M. Indicadores de Ciência, Tecnologia e Inovação no Brasil. Campinas: Ed. UNICAMP, 2003. p. 121-168.

LUUKKONEN, T. et al. The measurement of international scientific collaboration. Scientometrics, Amsterdam, v. 28, n. 1, p. 15-36, 1993.

MALTRÁS BARBA, B. Los indicadores bibliométricos: fundamentos y aplicación al análisis de la ciencia. Madrid: Trea, 2003. 
MARÇULA, M.; BENINI FILHO, P. A. Informática: conceitos e aplicações. São Paulo: Érica, 2005.

MEADOWS, J. A comunicação científica. Brasília, DF: Briquet de Lemos, 1999.

MOURA, A. M. M. et al. Panorama da produção conjunta entre Brasil e Espanha indexada na WoS entre 2006-2012. Informação \& Sociedade, João Pessoa, v. 25, n. 1, 2015.

SANTORO, A. F. S.; NOVAES, S. F. Física de altas energias: características e particularidades da área. Rio de Janeiro: Sociedade Brasileira de Física, 2003. Disponível em: <http://www.sbfisica.org.br/comissao_pc/HEPDOC.pdf>. Acesso em: 01 ago. 2015. SOCIEDADE BRASILEIRA DE FÍSICA - SBF. Relatório A Física e o desenvolvimento nacional. Brasília, D.F.: Centro de Gestão e Estudos Estratégicos, 2012.

VANZ, S. A. S. As redes de colaboração científica no Brasil: (2004-2006). 2009. 204 f. Tese (Doutorado) - Universidade Federal do Rio Grande do Sul, Faculdade de Biblioteconomia e Comunicação, Porto Alegre, 2009.

VANZ, S. A. S. et al. Scientific collaboration between Brazil and Spain: journals and citations. Encontros Bibli: revista eletrônica de biblioteconomia e ciência da Informação, Florianópolis, v. 21, n. 47, p. 41-50, set./dez., 2016.

VANZ, S. A. S; STUMPF, I. R. C. Colaboração científica: revisão teórico conceitual. Perspectivas em Ciência da Informação, Belo Horizonte, v. 15, n. 2, p. 42-55, maio/ago., 2010.

WAGNER, C. International collaboration in science and technology: promises and pitfalls. In: BOX, L.; ENGELHARD, R. (Ed.). Science and Technology Policy for Development, Dialogues at the Interface. London: Anthem Press, 2006. 